ORIGINAL PAPER

\title{
The value of BAP 1 immunocytochemistry and CDKN2A \\ FLUORESCENCE IN SITU HYBRIDIZATION IN DIAGNOSIS \\ OF SEROUS EFFUSION MALIGNANT MESOTHELIOMA AND \\ AN ANALYSIS OF THE ASSOCIATION BETWEEN DEGREE OF CELL ATYPIA AND THE RESULTS OF TWO AUXILIARY METHODS
}

\author{
Wenhao Ren ${ }^{1}$, Lin-lin ZhaO ${ }^{1}$, Lei Guo ${ }^{1}$, Ning Lu ${ }^{1}$, Jian CaO ${ }^{1,2}$
}

\begin{abstract}
${ }^{1}$ Department of Pathology and Resident Training Base, National Cancer Center/National Clinical Research Center for Cancer/Cancer Hospital, Chinese Academy of Medical Sciences and Peking Union Medical College, Beijing, China ${ }^{2}$ Department of Pathology, National Cancer Center/National Clinical Research Center for Cancer/Cancer Hospital and Shenzhen Hospital, Chinese Academy of Medical Sciences and Peking Union Medical College, Shenzhen, China
\end{abstract}

\begin{abstract}
Malignant mesothelioma (MM) is a rare, highly aggressive tumor. The first symptom of MM is mostly serous effusion, and cytology can be used in diagnosis based on effusion, providing patients with an earlier diagnosis and treatment opportunity. A total of 67 specimens were embedded into cell blocks, and BAP1 immunocytochemistry (ICC) was performed. CDKN2A fluorescence in situ hybridization (FISH) was performed in 45 cases. The sensitivity, specificity and the association between the degree of cell atypia and the results of two auxiliary methods were analyzed.

BAP1 ICC showed nonexpression in 13 of 24 cases of MM and 0 of 21 cases of benign mesothelial proliferation (BMP). The sensitivity was 54.2\% (13/24), and the specificity was $100 \%(21 / 21)$. In addition, 22 metastatic adenocarcinoma (MA) cases all showed BAP1 expression. MM with BAP1 expression had more obvious cell atypia. CDKN2A deletion was found in 12 of $24 \mathrm{MM}$ cases and 0 of $21 \mathrm{BMP}$ cases. The sensitivity was $50 \%(12 / 24)$, and the specificity was $100 \%(21 / 21)$. BAP1 ICC and CDKN2A FISH are useful methods to differentiate MM from BMP. The cell atypia of MM with BAP1 expression was more obvious than MM with BAP1 nonexpression.
\end{abstract}

Key words: malignant mesothelioma, immunocytochemistry, fluorescence in situ hybridization, BAP1, CDKN2A.

\section{Introduction}

Malignant mesothelioma (MM) is a highly invasive malignant tumor. Serous effusion is often the most common first symptom in MM, and mesothelioma cells often need to be differentiated from benign mesothelial proliferation (BMP) or metastatic adenocarcinoma (MA). Morphologically, their separation is often difficult. The most common genomic alterations were in $B A P 1, N F 2, C D K N 2 A / B$ and TP53 in patients with mesothelioma [1]. There is a high coincidence rate between BAP1 genomic alteration and immunohistochemistry, and fluorescence in situ hybridization (FISH) is the most suitable method to detect CDKN2A genomic alteration. BAP1 immunocytochemistry (ICC) and 
CDKN2A fluorescence in situ hybridization (FISH) were useful for the diagnosis of mesothelioma. Furthermore, the association between the degree of cell atypia and the results of the two auxiliary methods was worthy of analysis.
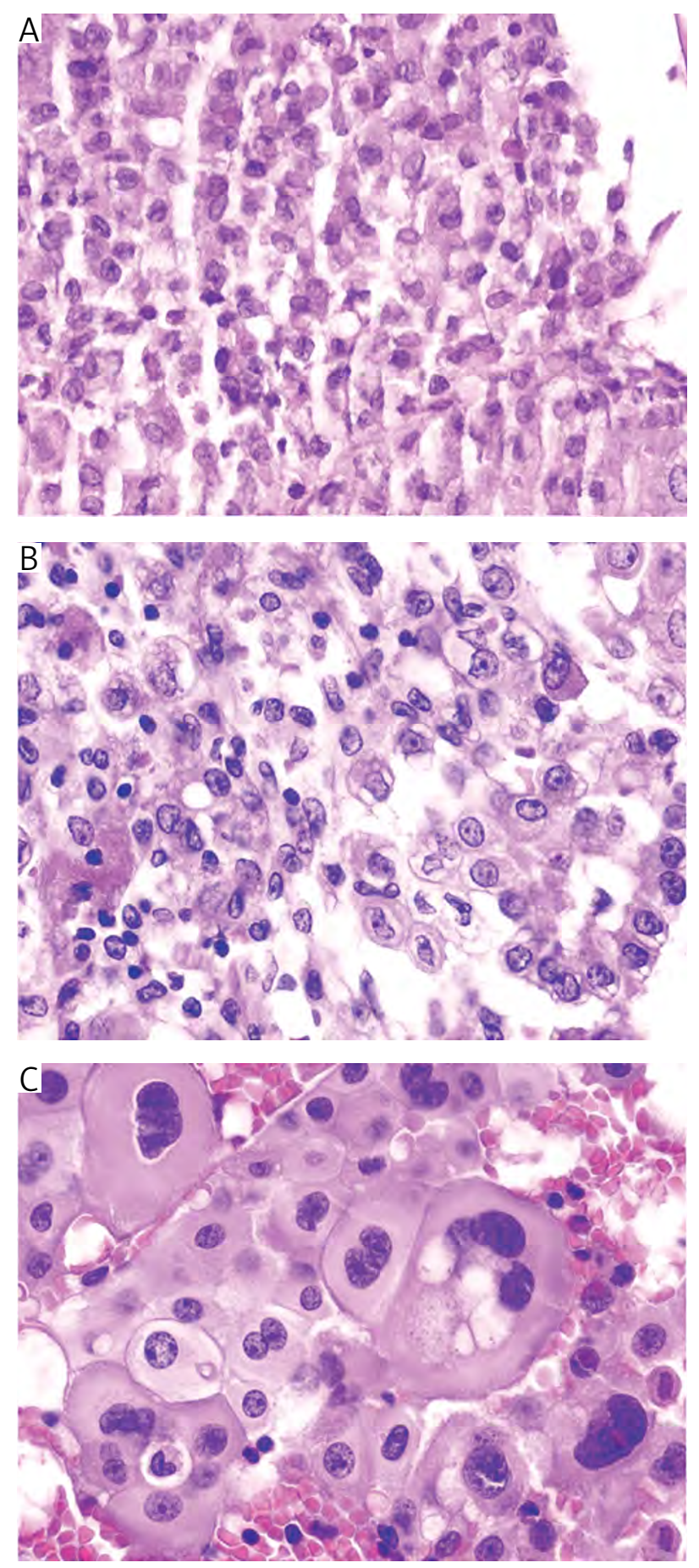

Fig. 1. Cell atypia of malignant mesothelioma (HE stain; original magnification $400 \times$ ). A) $1+$ (mild atypia): tumor cells showing mild nuclear atypia, fine granular chromatin, and indistinct nucleoli. B) 2+ (moderate atypia): tumor cells showing moderate nuclear atypia, and distinct nucleoli. C) $3+$ (severe atypia): tumor cells showing severe atypia, coarse granular chromatin)

\section{Material and methods}

\section{Immunocytochemistry}

Sixty-seven clinically redundant specimens of serous effusions from October 2015 to August 2017 were selected, including 46 pleural effusions, 19 ascites, and 2 pericardial effusions, with $24 \mathrm{MM}$ cases (20 malignant pleural mesothelioma and $4 \mathrm{ma}-$ lignant peritoneal mesothelioma), 21 BMP cases (14 benign pleural mesothelial proliferation, 5 benign peritoneal mesothelial proliferation, and 2 benign pericardial mesothelial proliferation) and 22 MA cases (11 lung adenocarcinoma and 11 ovarian cancer). Each sample was centrifuged and paraffin-embedded to prepare a cell block (plasma thrombin method). The cell block was sectioned (4- $\mu \mathrm{m}$ thick) and subjected to immunocytochemical staining of BAP1 (clone C-4; Santa Cruz Biotechnology, Santa Cruz, CA, USA; 1:200 dilution).

\section{Fluorescence in situ hybridization}

All 45 specimens for FISH were obtained from the clinically redundant specimens of serous effusions from October 2015 to August 2017, including 24 MM cases (20 malignant pleural mesothelioma and 4 malignant peritoneal mesothelioma) and 21 BMP cases (14 benign pleural mesothelial proliferation, 5 benign peritoneal mesothelial proliferation, and 2 benign pericardial mesothelial proliferation). A cell block (plasma thrombin method) was prepared in each case, and the cell block was sectioned ( $4-\mu \mathrm{m}$ thick) and examined by FISH for CDKN2A (Vysis CDKN2A/CEP 9 FISH Probe Kit using TermoBrite in situ hybridization instrument Abbott, USA).

Patients with MM and MA were all histologically and clinically confirmed. Patients with reactive effusions were followed for a median of 24 months (range 12-35 months), and none developed MM. Clinical diagnoses included tuberculous peritonitis, primary mediastinal large B-cell lymphoma, meningioma and histologically confirmed metastatic carcinoma.

\section{Positive signal determination}

\section{Immunocytochemistry}

Nonmesothelial BAP1-reactive cells, such as tissue cells, fibroblasts, inflammatory cells, and endothelial cells, served as internal positive controls in each section. Nuclear staining was regarded as a positive result, and cytoplasmic staining was regarded as nonspecific staining. 


\section{Fluorescence in situ bybridization}

The CDKN2A FISH probe is a CDKN2A/CEP 9 double-labeled probe (red-labeled CDKN2A fragment, green-labeled centromere). A homozygous deletion is defined as a CDKN2A double gene deletion, and a heterozygous deletion is defined as one CDKN2A gene deletion. FISH results were defined as "positive" for the diagnosis of MM when a CDKN2A(p16) homozygous deletion pattern was observed in $>15 \%$ of the atypical mesothelial cells analyzed or a CDKN2A(p16) heterozygous deletion pattern was observed in $>41.5 \%$ of atypical mesothelial cells analyzed.

\section{Degree of cell atypia of malignant mesothelioma}

Cell atypia was classified according to the degree: $1+$ (mild atypia): nuclei uniform in size and shape, indistinct nucleoli, fine granular chromatin (Fig. 1A); $2+$ (moderate atypia): nuclei of intermediate size between mild and severe, with slight irregularity in shape, distinct nucleoli (Fig. 1B); and 3+ (severe atypia): bizarre, enlarged nuclei of varied sizes, with some nuclei at least twice as large as others, coarse granular chromatin, large nucleoli (Fig. 1C). Prominence of nucleoli was evaluated using as a reference nearby red blood cells, which measured approximately $7 \mu \mathrm{m}$, and graded as the following three categories by the measurement of predominant size: 1) indistinct: inconspicuous or very small (Fig. 1A); 2) distinct: $<3 \mu \mathrm{m}$ (Fig. 1B); 3) large $\geq 3 \mu \mathrm{m}$ (Fig. 1C). All slides were reviewed by two pathologists. Cell features were evaluated using a high-power field (HPF) at $\times 400$ magnification using an Olympus BX43 microscope (Olympus, Tokyo, Japan). Cell atypia was recorded only if it represented $>5 \%$ of the entire tumor area.

\section{Statistical analysis}

Statistically analysis was performed using the SPSS 19.0 statistical software package. The Mann-Whitney test and $\chi^{2}$ test were performed. A p-value $<0.05$ was considered a statistically significant result.

\section{Results}

\section{Clinical features}

The mean age was 34.5 years (range, 18-78 years). The mean age of patients in the MM group was 65.5 years (range, $42-85$ years), and the male-tofemale ratio was $2: 1$. The mean age of patients in the BMP group was 58.8 years (range, 25-78 years). The male-to-female ratio was $1.3: 1$. The average age of patients in the MA group was 58.2 years (range, 45-76 years). The male-to-female ratio was 1.2:1.

\section{Expression of BAP1 immunocytochemistry in serous effusion specimens}

BAP1 was not expressed in 13 of $24 \mathrm{MM}$ cases and 0 of $21 \mathrm{BPM}$ cases. The sensitivity to MM was $54.2 \%$ (13/24), and the specificity was $100 \%$ (21/21). After the $\chi^{2}$ test, the expression of BAP1 in MM and BMP was significantly different $\left(\chi^{2}=62.4, \mathrm{p}<0.005\right)$. In addition, BAP1 was not expressed in 0 of $22 \mathrm{MA}$ cases (Fig. 2).

The results of BAP1 ICC and the corresponding atypia degree are shown in Table I. Two independent sample nonparametric tests (Mann-Whitney test) were performed on the expression of BAP1 ICC and the degree of cell atypia. There was a significant difference between BAP1 expression and the degree of cell atypia ( $p=0.030<0.05)$; the cell atypia of MM with BAP1 expression was more obvious.

\section{Expression of CDKN2A in serous effusion specimens}

Of the 21 BMP patients, 0 had a CDKN2A gene deletion (Fig. 3). Twelve of the $24 \mathrm{MM}$ patients had a CDKN2A gene deletion (Fig. 4), 11 showed a homozygous deletion pattern, and 1 exhibited a heterozygous deletion pattern. The sensitivity to $\mathrm{MM}$ was $50 \%(12 / 24)$, and the specificity was $100 \%(21 / 21)$. After the $\chi^{2}$ test, the deletion of CDKN2A in MM and BMP was significantly different $\left(\chi^{2}=60.6\right.$, $\mathrm{p}<0.005)$.

The results of CDKN2A FISH and the corresponding atypia degree are shown in Table I. Two independent sample nonparametric tests (Mann-Whitney test) were performed on the deletion of CDKN2A and the degree of cell atypia, and the cell atypia showed no significant difference between CDKN2A-deletion and nondeletion MM ( $p=0.29>0.05)$.

\section{Value of CDKN2A FISH combined with BAP1 ICC in serous effusion specimens}

When combining CDKN2A FISH and BAP1 ICC as a panel, of the $24 \mathrm{MM}$ cases, 19 showed either BAP1 nonexpression or CDKN2A deletion (19/24 [79.2\%]). BAP1 nonexpression and CDKN2A deletion was not found in any of the 21 benign cases $(0 / 21[0 \%])$. Six cases of mesothelioma showed both BAP1 nonexpression and CDKN2A deletion (6/24 [25\%]) (Fig. 5).

The nonexpression rate of BAP1 in malignant pleural mesothelioma was 50\% (10/20), and the nonexpression rate in malignant peritoneal mesothelioma was $75 \%(3 / 4)$. The deletion rate of CDKN2A in malignant pleural mesothelioma was 55\% (11/20), and the deletion rate in malignant peritoneal mesothelioma was $25 \%(1 / 4)$. 

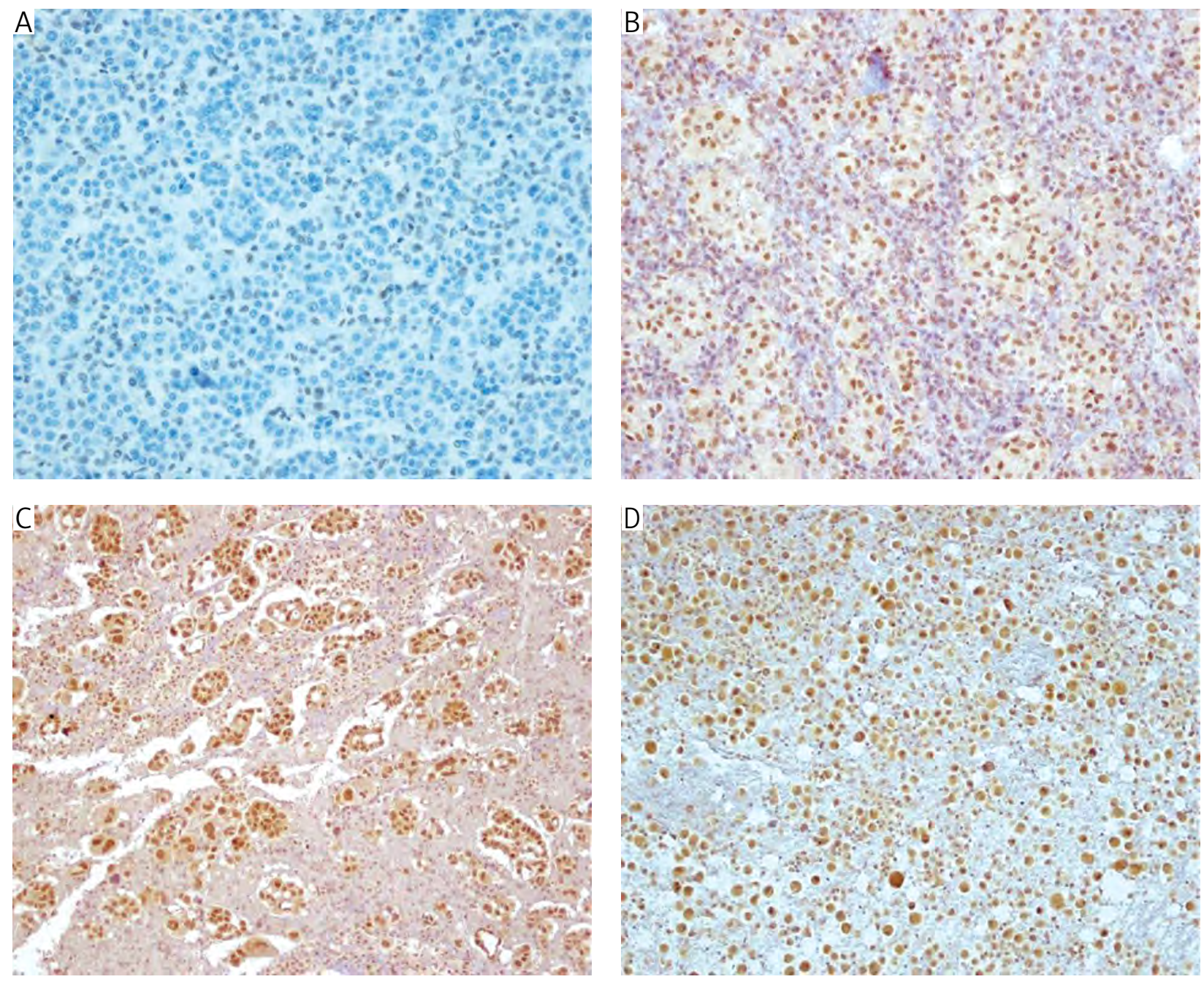

Fig. 2. The expression of BAP1 immunocytochemistry (ICC) in serous effusion specimens. BAP1 shows loss of nuclear immunoreactivity in malignant mesothelioma (A), while nuclear immunoreactivity exists in benign mesothelial proliferation (B), metastatic lung adenocarcinoma (C) and metastatic ovarian cancer (D)

\section{Discussion}

The most common malignant tumors of serous effusion are MM and MA; BMP needs to be differentiated. The morphology of BMP, MA and MM has a large overlap, so the morphological diagnosis of MM is a difficult problem in cytopathological diagnosis. ICC is an important method of differential diagnosis. It is relatively easy to distinguish MM from MA by ICC, but it is difficult to distinguish BMP from MM. BAP1 is a useful antibody to distinguish MM from BMP. The BAP1 gene is located on the human chromosome 3p21, and epidemiological studies have shown that mutations in the BAP1 gene cause a high incidence of MM in families without occupational exposure to asbestos $[2,3]$. Protein expression is lost when BAP1 has a biallelic mutation. Recently, some studies have reported that BAP1 nuclear staining can identify MM and BMP; the former is usually negative, the latter BAP1 is usually positive $[4,5,6,7]$. In ad- dition, the nonexpression rate of BAP1 in epithelioid MM is $56-81 \%[7,8,9,10,11]$, and the rate of nonexpression in sarcomatoid MM is lower [12], while the rate of BAP1 nonexpression in BMP is $0 \%[8,9$, $10,12,13]$. BAP1 is also usually expressed in lung adenocarcinoma and ovarian serous carcinoma [12]. In this study, we performed BAP1 ICC on $67 \mathrm{spec}-$ imens. The nonexpression rate of BAP1 in MM was $54.2 \%$ (13/24), which was slightly lower than that reported in the literature. The nonexpression rate in malignant pleural mesothelioma was 50\% (10/20), while in malignant peritoneal mesothelioma it was $75 \%$ (3/4); the rate of nonexpression in BMP was $0 \%$ $(0 / 21)$, while in MA it was $0 \%(0 / 22)$. In metastatic lung adenocarcinoma it was $0 \%(0 / 11)$ and in metastatic ovarian cancer it was $0 \%(0 / 11)$. This reminds us that in daily clinical work, if the BAP1 ICC does not show staining in the nucleus, it prompts a diagnosis of MM. If BAP1 is expressed, MM cannot be excluded. The expression of BAP1 in some MM 
Table I. Results of BAP1 ICC and CDKN2A FISH and corresponding atypia degree

\begin{tabular}{|c|c|c|c|c|}
\hline CASE & SPECIMEN TYPE & BAP1 & CDKN2A & ATYPIA DEGREE \\
\hline 1 & Pleural effusion & Nonexpression & Deletion & 2 \\
\hline 2 & Pleural effusion & Nonexpression & Nondeletion & 2 \\
\hline 3 & Pleural effusion & Nonexpression & Deletion & 3 \\
\hline 4 & Pleural effusion & Expression & Deletion & 3 \\
\hline 5 & Pleural effusion & Expression & Nondeletion & 2 \\
\hline 6 & Pleural effusion & Nonexpression & Deletion & 1 \\
\hline 7 & Pleural effusion & Expression & Deletion & 3 \\
\hline 8 & Pleural effusion & Expression & Deletion & 3 \\
\hline 9 & Pleural effusion & Nonexpression & Nondeletion & 2 \\
\hline 10 & Pleural effusion & Nonexpression & Deletion & 3 \\
\hline 11 & Pleural effusion & Nonexpression & Nondeletion & 1 \\
\hline 12 & Pleural effusion & Expression & Nondeletion & 3 \\
\hline 13 & Pleural effusion & Expression & Deletion & 3 \\
\hline 14 & Pleural effusion & Expression & Nondeletion & 1 \\
\hline 15 & Pleural effusion & Expression & Deletion & 3 \\
\hline 16 & Pleural effusion & Nonexpression & Nondeletion & 3 \\
\hline 17 & Pleural effusion & Expression & Nondeletion & 3 \\
\hline 18 & Pleural effusion & Nonexpression & Nondeletion & 1 \\
\hline 19 & Pleural effusion & Nonexpression & Deletion & 2 \\
\hline 20 & Pleural effusion & Expression & Deletion & 3 \\
\hline 21 & Ascites & Nonexpression & Nondeletion & 3 \\
\hline 22 & Ascites & Nonexpression & Nondeletion & 1 \\
\hline 23 & Ascites & Nonexpression & Deletion & 1 \\
\hline 24 & Ascites & Expression & Nondeletion & 3 \\
\hline
\end{tabular}

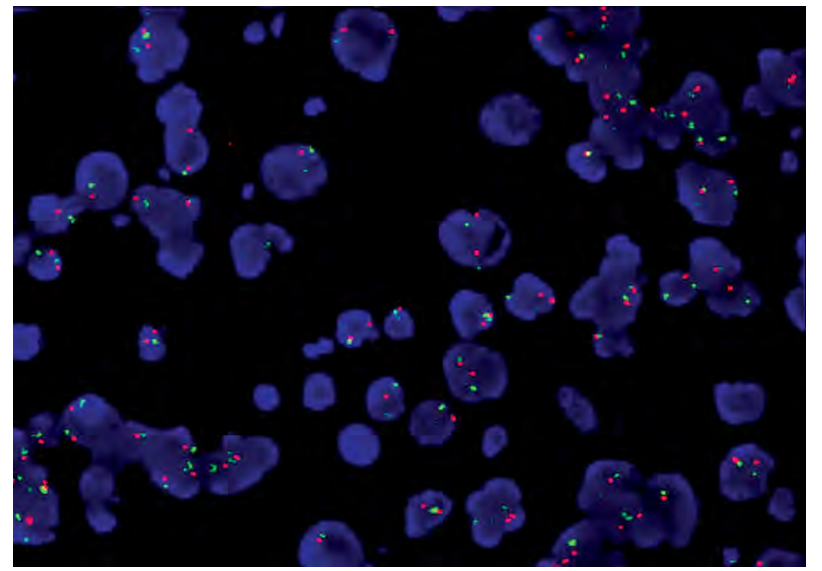

Fig. 3. Fluorescence in situ hybridization (FISH) of CDKN2A in benign mesothelial proliferation specimens shows that centromere (green) and double CDKN2A fragments (red) are present

cases may be related to the fact that only double allele mutations of the BAP1 gene lead to protein nonexpression. We also found that the cell atypia

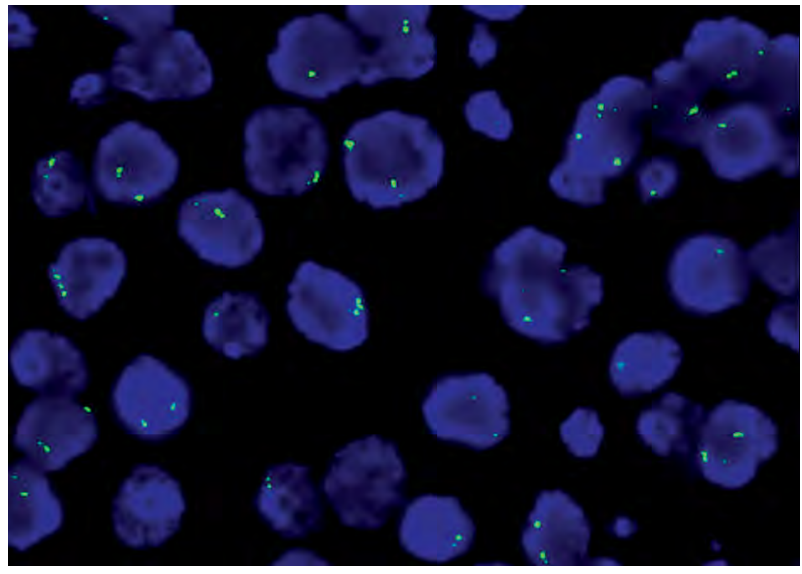

Fig. 4. Fluorescence in situ hybridization (FISH) of CDKN2A in malignant mesothelioma specimens shows that the centromere (green) was still present, but the CDKN2A fragment (red) was deleted

of MM with BAP1 expression is more obvious, BAP1 nonexpression is not only evidence for the diagnosis of mesothelioma, but also the cell atypia of MM with 


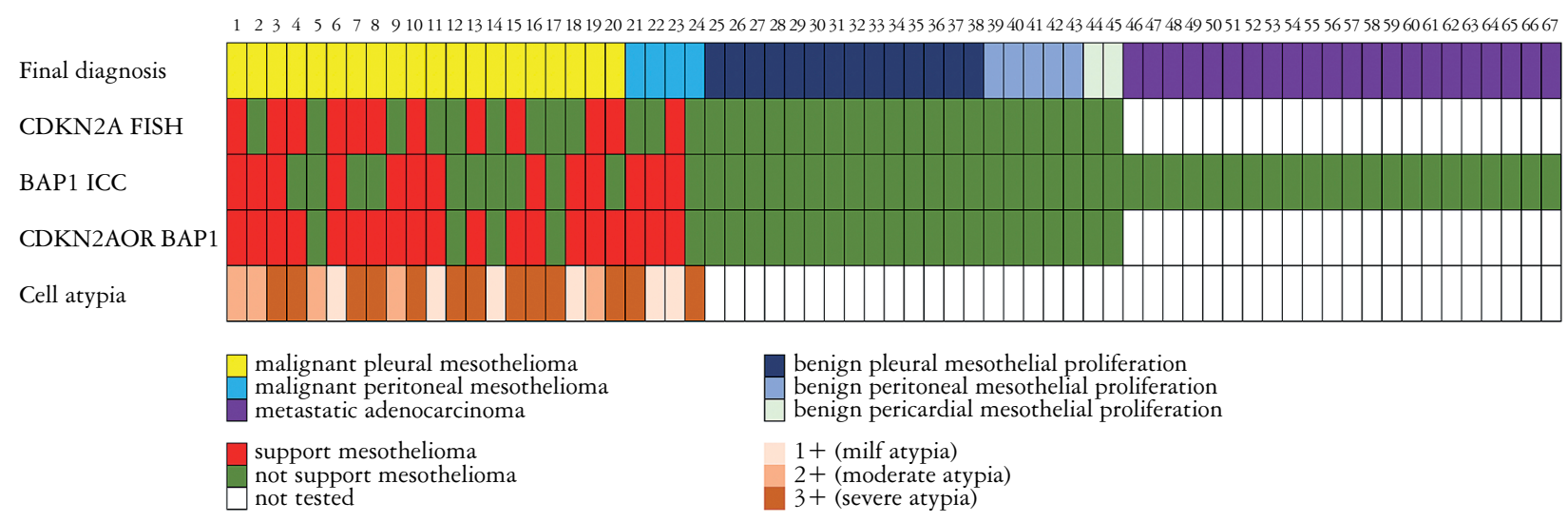

Fig. 5. An overview of BAP1 ICC, CDKN2A FISH and cell atypia in serous effusion specimens

BAP1 nonexpression is smaller, which will be useful for the diagnosis of MM when cell atypia is small. The relationship between cell atypia and BAP1 status is not reported in the literature, but some studies reveal that the prognosis of MM with BAP1 nonexpression is better $[8,14]$. To some extent, they were consistent with our study, where more obvious cell atypia suggested a worse prognosis.

Another important finding in $\mathrm{MM}$ is the deletion of chromosome 9p21, which contains the CDKN2A, CDKN2B and methylthioadenosine phosphorylase (MTAP) genes. P16/CDKN2A deletion only occurs in MM, while point mutation and DNA methylation can be seen in BMP, so detection of P16/CDKN2A contributes to the differential diagnosis of BMP and MM. FISH is currently considered to be the most appropriate method for detecting CDKN2A deletion. The literature reports that the deletion rate of CDKN2A in pleural epithelioid MM is $45-86 \%$ $[15,16,17,18,19]$; the deletion rate in peritoneal epithelioid MM is $14-51 \%$ [15, 16, 18, 20, 21]. The highest expression rate was in sarcomatoid MM with 50-100\% $[13,22,23,24]$, and the deletion rate in $\mathrm{BMP}$ is $0 \%[11,15,17,18,19,21,23]$. In this study, the deletion rate of CDKN2A in MM was $50 \%(12 / 24)$, the deletion rate in peritoneal MM was $25 \%(1 / 4)$, and the deletion rate in pleural MM was $55 \%(11 / 20)$, which is consistent with the literature report. The literature reports that patients with CDKN2A deficiency have a poor prognosis [16]. However, in our study, no significant difference in atypia between CDKN2A deleted and nondeleted $\mathrm{MM}$ was found.

BAP1 ICC and CDKN2A FISH have different positive rates in different subtypes of MM (epithelioid, sarcomatoid or mixed) and different parts (pleura and peritoneum); therefore, the combined use of BAP1 ICC and CDKN2A FISH can theoretically improve the diagnostic rate of MM. In this study, BAP1 ICC staining combined with CDKN2A FISH improved the diagnostic rate to $79.2 \%(19 / 24)$, and
BAP1 ICC combined with CDKN2A FISH is an effective diagnostic method.

Another aspect of this study is to analyze the association between the degree of cell atypia and the results of two auxiliary methods. Cytopathologists often consider the need of auxiliary methods based on morphology to support the diagnosis. Some mesothelioma with mild morphology may not conduct immunohistochemistry for misdiagnosing as benign mesothelial proliferation, while they will choose immunohistochemistry for mesothelioma whose cell atypia is severe, but the results of BAP1 immunohistochemistry are often unsatisfactory for this kind of mesothelioma, which may lead to the gradual abandonment of BAP1 antibody. Our study can improve the above situation, making the application of BAP1 immunohistochemistry more accurate. We found that BAP1 immunohistochemistry is more easily expressed in mesothelioma with severe atypia, and BAP1 immunohistochemistry is more suitable for supporting the diagnosis of mesothelioma whose morphology is moderate. We also analyzed the relationship between the degree of cell atypia and CDKN2A FISH, but the results showed that the cell atypia showed no significant difference between CDKN2A-deleted and nondeleted mesothelioma. In clinical application, if the tumor with severe atypia needs the support of auxiliary methods and BAP1 immunohistochemistry does not work, CDKN2A FISH can be chosen.

However, this study also has many shortcomings. First, the sample size of the study is small, and a larger sample would make our results more reliable. Second, most MM cells in serous effusion come from epithelioid or mixed MM, and few sarcomatoid MM cells are found in serous effusion. Therefore, the results of this study can only represent some features of epithelioid MM and mixed MM. Third, because the changes in the BAP1 gene are in agreement with the expression of BAP1 protein, we only examined the expression of BAP1 protein by ICC, but 
the expression of the BAP1 protein cannot completely represent the changes in the BAP1 gene. Fourth, because all the samples in the study were collected in recent years, there was no survival analysis for the patients in the study.

In conclusion, BAP1 ICC and CDKN2A FISH have independent diagnostic value for the diagnosis and differential diagnosis of MM. The combination of the results of BAP1 ICC and CDKN2A FISH increases the sensitivity and accuracy. These results will contribute to the early diagnosis and correct treatment of MM. Combining these diagnostic procedures with other ICC projects, such as calretinin, D2-40, WT- 1 and CK5/6, which are widely used in clinical practice, will make the diagnosis of MM more accurate. Other genes that are frequently mutated in MM, such as NF2, CUL1, and EWSR1, still need more economical and effective methods for the diagnosis of MM. The diagnosis of MM still needs additional study.

Acknowledgment of grant support: this research was performed with grant support from Sanming Project of Medicine in Shenzhen, Grant/Award Number: SZSM201812076.

The authors declare no conflict of interest.

\section{References}

1. Kato S, Tomson BN, Buys TP, et al. Genomic Landscape of Malignant Mesotheliomas. Mol Cancer Ther 2016; 15: 2498-2507.

2. Testa JR, Cheung M, Pei J, et al. Germline BAP1 mutations predispose to malignant mesothelioma. Nat Genet 2011; 43 : 1022-1025.

3. Carbone M, Flores EG, Emi M, et al. Combined genetic and genealogic studies uncover a large BAP1 cancer syndrome kindred tracing back nine generations to a common ancestor from the 1700s. PLoS Genet 2015; 11: e1005633.

4. Andrici J, Jung J, Sheen A, et al. Loss of BAP1 expression is very rare in peritoneal and gynecologic serous adenocarcinomas and can be useful in the differential diagnosis with abdominal mesothelioma. Hum Pathol 2016; 51: 9-15.

5. Hwang HC, Pyott S, Rodriguez S, et al. BAP1 immunohistochemistry and p16 FISH in the diagnosis of sarcomatous and desmoplastic mesotheliomas. Am J Surg Pathol 2016; 40: 714-718.

6. Andrici J, Sheen A, Sioson L, et al. Loss of expression of BAP1 is a useful adjunct, which strongly supports the diagnosis of mesothelioma in effusion cytology. Mod Pathol 2015; 28: 1360-1368.

7. Cigognetti M, Lonardi S, Fisogni S, et al. BAP1 (BRCA1-associated protein 1) is a highly specific marker for differentiating mesothelioma from reactive mesothelial proliferations. Mod Pathol 2015; 28: 1043-1057.

8. Farzin M, Toon CW, Clarkson A, et al. Loss of expression of BAP1 predicts longer survival in mesothelioma. Pathology 2015; 47: 302-307.

9. Nasu M, Emi M, Pastorino S, et al. High incidence of somatic BAP1 alterations in sporadic malignant mesothelioma. J Thorac Oncol 2015; 10: 565-576.
10. Yoshikawa Y, Sato A, Tsujimura T, et al. Frequent inactivation of the BAP1 gene in epithelioid-type malignant mesothelioma. Cancer Sci 2012; 103: 868-874.

11. Sheffield BS, Hwang HC, Lee AF, et al. BAP1 immunohistochemistry and p16 FISH to separate benign from malignant mesothelial proliferations. Am J Surg Pathol 2015; 39: 977-982.

12. Cigognetti M, Lonardi S, Fisogni S, et al. BAP1 (BRCA1-associated protein 1 ) is a highly specific marker for differentiating mesothelioma from reactive mesothelial proliferations. Mod Pathol 2015; 28: 1043-1057.

13. Sheffield BS, Hwang HC, Lee AF, et al. BAP1 immunohistochemistry and p16 FISH to separate benign from malignant mesothelial proliferations. Am J Surg Pathol 2015; 39: 977-982.

14. Mcgregor SM, Dunning R, Hyjek E, et al. BAP1 facilitates diagnostic objectivity, classification, and prognostication in malignant pleural mesothelioma. Hum Pathol 2015; 46: 1670-1678.

15. Monaco SE, Shuai Y, Bansal M, et al. The diagnostic utility of p16 FISH and GLUT-1 immunohistochemical analysis in mesothelial proliferations. Am J Clin Pathol 2011; 135: 619-627.

16. Krasinskas AM, Bartlett DL, Cieply K, et al. CDKN2A and MTAP deletions in peritoneal mesotheliomas are correlated with loss of p16 protein expression and poor survival. Mod Pathol 2010; 23: 531-538.

17. Illei PB, Rusch VW, Zakowski MF, et al. Homozygous deletion of CDKN2A and codeletion of the methylthioadenosine phosphorylase gene in the majority of pleural mesotheliomas. Clin Cancer Res 2003; 9: 2108-2113.

18. Chiosea S, Krasinskas A, Cagle PT, et al. Diagnostic importance of 9p21 homozygous deletion in malignant mesotheliomas. Mod Pathol 2008; 21: 742-747.

19. Chung CT, Santos GC, Hwang DM, et al. FISH assay development for the detection of $\mathrm{p} 16 / \mathrm{CDKN} 2 \mathrm{~A}$ deletion in malignant pleural mesothelioma. J Clin Pathol 2010; 63: 630-634.

20. Hwang H, Tse C, Rodriguez S, et al. p16 FISH deletion in surface epithelial mesothelial proliferations is predictive of underlying invasive mesothelioma. Am J Surg Pathol 2014; 38: 681-688.

21. Ito T, Hamasaki M, Matsumoto S, et al. p16/CDKN2A FISH in differentiation of diffuse malignant peritoneal mesothelioma from mesothelial hyperplasia and epithelial ovarian cancer. Am J Clin Pathol 2015; 143: 830-838.

22. Tochigi N, Attanoos R, Chirieac LR, et al. p16 Deletion in sarcomatoid tumors of the lung and pleura. Arch Pathol Lab Med 2013; 137: 632-636.

23. Takeda M, Kasai T, Enomoto Y, et al. 9p21 deletion in the diagnosis of malignant mesothelioma, using fluorescence in situ hybridization analysis. Pathol Int 2010; 60: 395-399.

24. Wu D, Hiroshima K, Matsumoto S, et al. Diagnostic usefulness of p16/CDKN2A FISH in distinguishing between sarcomatoid mesothelioma and fibrous pleuritis. Am J Clin Pathol 2013; 139: 39-46.

\section{Address for correspondence}

\section{Jian Cao}

Department of Pathology

National Cancer Center/

National Clinical Research Center for Cancer/

Cancer Hospital and Shenzhen Hospital

Chinese Academy of Medical Sciences

and Peking Union Medical College

Shenzhen, China

e-mail: caojian828@163.com 